\title{
Informing Systems in Business Environments: A Purpose-Focused View
}

\author{
Zbigniew J Gackowski \\ California State University Stanislaus, Turlock, CA, USA
}

\author{
zgacko@athena.csustan.edu
}

\begin{abstract}
This paper presents a technology-independent rational inquiry into informing systems in business environments. Depending on the primary concerns, informing systems should be examined from either the viewpoint of information disseminators or informing clients. The latter viewpoint is subject to extensive empirical studies within informing science and partially within the MIT Information Quality Program. It focuses mainly on information products, services, users' preferences, and requirement specifications. The information disseminators' viewpoint is rarely taken into account. Based on a short review of the most popular MIS textbooks and research in this domain, this paper discusses problems one encounters during examination of informing systems in business environments. It uses an improved version of the purpose-focused framework (Gackowski, 2004a), which covers both viewpoints. Two refinements of the Informing Science Framework as defined by Cohen (1999) are suggested.
\end{abstract}

Keywords: Informing schema, informing systems, information in decision situations, valid information, misinformation, disinformation, purpose-focused view on quality, operations research (OR) approach to data/information quality, quality requirements; information effective usability, usefulness, and economic usefulness; refinements to the Informing Science Framework.

\section{Introduction}

"The purpose of Informing Science is to encourage the sharing of knowledge and collaboration among the wide variety of fields that use information technology to inform clients" (INSITE 2004). Informing systems should be cost effective. In mission critical applications, their systematic examination should be conducted from either the viewpoint of information users - the clients or information disseminators, whichever matters more. The examination should be performed for all the identified factors that are of operationally and potentially significant impact.

Current MIS textbooks are deficient with regard to the role of end users and even more so about

Material published as part of this journal, either on-line or in print, is copyrighted by the publisher of the Informing Science Journal. Permission to make digital or paper copy of part or all of these works for personal or classroom use is granted without fee provided that the copies are not made or distributed for profit or commercial advantage AND that copies 1) bear this notice in full and 2) give the full citation on the first page. It is permissible to abstract these works so long as credit is given. To copy in all other cases or to republish or to post on a server or to redistribute to lists requires specific permission and payment of a fee. Contact Editor@inform.nu to request redistribution permission. the information disseminators. The texts are overly technology laden, with oversimplified coverage of the fundamentals on data, information, and particularly the role of informing in business. A summary of research published by Huang et al., (1999 p. 4) supports this statement in the following manner: "Many best-practice reports witness that information technology alone is not the driver for 
knowledge management in companies today. Information and knowledge experienced by members of an organization should be the focus, not the system or technology per se. ... Technology and systems are facilitators."

This paper points out the lack of adequate exposure of business students to examining and analyzing informing systems that they certainly will face in their professional career. This analysis is not a technical task for IT personnel; it is a task mainly for information disseminators and informing clients. To address this gap a technology-independent rational inquiry into informing systems is presented. This inquiry focuses mainly on informing in business environments, where in more or less free-market conditions, business organizations operate. Depending on the primary concerns of the situation, informing systems need to be examined from either the viewpoint of information disseminators or information users. In Informing Science, the latter are referred to as informing clients. The clients' viewpoint is subject to extensive empirical studies within informing science and partially within the MIT Information Quality Program, where the principles of Total Quality Management (TQM) are broadly used (see the AIMQ methodology (Lee et al., 2002). This approach focuses on information products, services, users' preferences, and requirement specifications. On the other hand, the information disseminators' viewpoint is less emphasized or even ignored. This inquiry is based on an operations research approach - the modified purpose-focused framework for assessing information quality as presented at the $9^{\text {th }}$ International Conference on Information Quality at MIT (Gackowski, 2004a). The paper consists of two major parts: (1) A short examination of the most popular MIS textbooks and the pertinent research in this domain, and (2) An outline of problems one encounters during examination of informing systems. It offers also a rationale for two modifications of the Informing Science Framework defined by Cohen (1999).

\section{Literature Review}

\section{What do the most popular textbooks say?}

No author mentions the art and science of informing. The concept of informing systems is not discussed. Most authors mention some quality attributes of data/information in an eclectic manner. The best our MIS students can find are piecemeal references to some quality attributes of data/information. Usually they are discussed in a sequence with no underlying logical structure.

O'Brien $(2003,2004$ p. 15 - 16), the author of the two most popular textbooks on MIS as judged by the number of published editions, defines "Information as data placed in a meaningful and useful context" (glossary), and "Information quality as the degree to which information has content, form, and time characteristics that give it value to specific end users." He suggests an examination of 15 attributes of quality of information within a three-dimensional framework: (1) time, (2) content, and (3) form, with short comments, but with no reference to the informing processes or systems. He mentions information value, but only from the end users', never the disseminators' viewpoint, and he does not define it.

It is ironic that Alter (1, p. 162-8) describes the fundamental concept of information economics the utility value of information with its simple and pragmatic definition as "more elegant than practical." He distinguishes four main factors of information usefulness: information quality, accessibility, presentation, and security, subdivided into characteristics that are more specific.

There are other examples. Malaga (2005, p. 8) suggests considering six qualities of information in the following sequence: accuracy, timeliness, accessibility, engagement, application (relevant), and rarity, Dock and Wetherbe (1988) suggest another six: accessibility, timeliness, rele- 
vance, accuracy, verifiability, completeness, and clarity. In summary, there is not much on the market about informing that serves MIS students well.

\section{What Does Research Offer?}

There is research on informing but not much of it is of general nature. The closest research of this type pertains to data or information quality. On one hand, based on an empirical definition of quality derived from the information consumer's perspective in (Wang \& Strong, 1996), Lee, Strong, Kahn, Wang developed AIMQ: A Methodology for Information Quality Assessment (2002). It focuses mainly on information products, services, users' preferences, and requirement specifications. On the other hand, Gackowski (2004a) proposed a purpose-focused perspective of assessing quality.

There is also research that focuses on intrinsic quality requirements: the ontological approach to quality of mapping real-world states into information system states by Wand and Wang (1996), and the quality requirement to databases summarized by Nauman and Roth (2004). They are important; however, they are limited mainly to the design of informing delivery systems.

\section{AIMQ: A methodology for information quality assessment}

Here the data/information quality is viewed from the information consumer's perspective, and based on the Total Quality Management (TQM) principles (Lee et al., 2002). Hence, information quality is not defined by providers or custodians of information, but instead, by information consumers. "Information quality" is defined as "information fit for use" by information consumers. It is treated as a product. The first essential component of the methodology is the PSP/IQ model, which considers four situations derived from the combination of two factors: whether one deals with an information product or information service, and whether one tries to meet specifications or expectations of information users.

It is a model within the confines of TQM principles; however, one should not ignore its inherent limitations. It is limited to products or services, and to given specifications or preferences of information users. These limitations are substantial when one realizes at least some of their consequences: (1) Products or services are not identical with purposes, goals, and objectives in business. (2) Specifications provided by a contracting entity may be sacred to a contractor, but they may be substantially deficient in meeting the actual business purpose. (3) Preferences of information users within a business entity may deviate considerably from or even be in conflict with business purposes.

\section{PFV/IQ The purpose-focused data/information quality assessment}

The teleological operations research-based purpose-focused view on data/information quality proposed by Gackowski (2004a) for business environment is a radical departure from the prevalent TQM based methodologies and survey-based empirical studies from the subjective end users' perspective. It concentrates on assessing the quality of all aspects of informing by the cost effectiveness of obtaining well-defined results that are considered the main business purpose.

It is anchored in the concept of business decision situations. It fully recognizes and acknowledges the importance of the ontological foundations of data/information quality, but it emphasizes the other side of quality that is anchored in the teleological foundations of operations research, management science, and decision science. This view should be the dominant one, in business, public administration, and military operations. 
Informing Systems in Business Environments

PFV/IQ offers a sound basis for a hierarchical result-oriented taxonomy of the entire universe of dimensions of data/information quality whether viewed by disseminators or end users (clients). An insight into the logical interdependencies among those dimensions leads to a natural and economical sequence of their examination. It accommodates and complements the accumulated findings. In addition, it provides analysts with a situational reference point for ranking data or information values by their impact on the business results to determine which should be examined first, and relatively how much attention pay to each of them.

\section{Informing Systems - A Purpose-Focused View}

\section{Basics about Informing}

It seems that in English literature, the first formal definition of informing systems within the realm of information science can be found in "An Approach to Categorization of Information Systems" by Gackowski (1982). Within the context of Nadler's (1970) terminology on work systems, informing systems are defined there as a class of work systems whose basic output is information that affects the actions of its recipients.

Any informing model consists of at least three basic components: information source(s), communication channel(s), and information receiving entities, the last of which are referred to as informing clients within the realm of informing science.

Information sources may be active or passive.

- Active information sources on their own emit, transmit, disseminate, or broadcast signals conveying information; it may happen by their nature or by design. Any active source emits by its nature signals that may convey information. When by design, the source is the main active agent, a sender, or disseminator of information who tries to provide some kind of information service for the clients or affect them in a desired manner. One can mention here at least: advertisers, professional information providers, politicians, preachers, etc.

- Passive information sources provide signals conveying information in the process of observing, examining, and/or measuring them.

Communications channels link information sources with information receiving clients. The entire set and sequence of transforming those signals constitutes the informing process. In most cases, the informing processes are done by the sources, the communication channels and by the clients. Most of the processes are performed by delivery systems, which are among the three main components of the informing science framework.

Informing may be direct or indirect.

- Direct informing takes place when signals conveying information flow directly between information sources and clients.

- Indirect informing takes place when intermediary storing and processing of information takes place between information sources and clients. In an advanced environment, data are usually organized in databases or data warehouses. These may be run by information providers or by client organizations for themselves.

Similarly, informing may be solicited or unsolicited by clients.

Informing clients must be capable of acting autonomously so that the incoming information may cause behave them differently than without the received information. Clients may be simple or 
composite entities, individuals and/or organizations, or even robotic devices, usually numerically controlled ones. Within a client's entity a division of labor may take place that results in specialized sub-entities that deal with information acquisition, its presentation, decision-making, and implementation of the decisions made - the actually acting agents. Examples of autonomous clients are marketing prospects, competitors, adversaries, students, voters, the public, etc.

In general, clients may be intentionally targeted by information sources or they may actively gather information from any sources, thus they may be interested in being provided with some information products or services. Clients may pay for being informed or seek only information offered seemingly free. They may also to be inclined to enter into a dialog to refine the informing or refuse to participate in it.

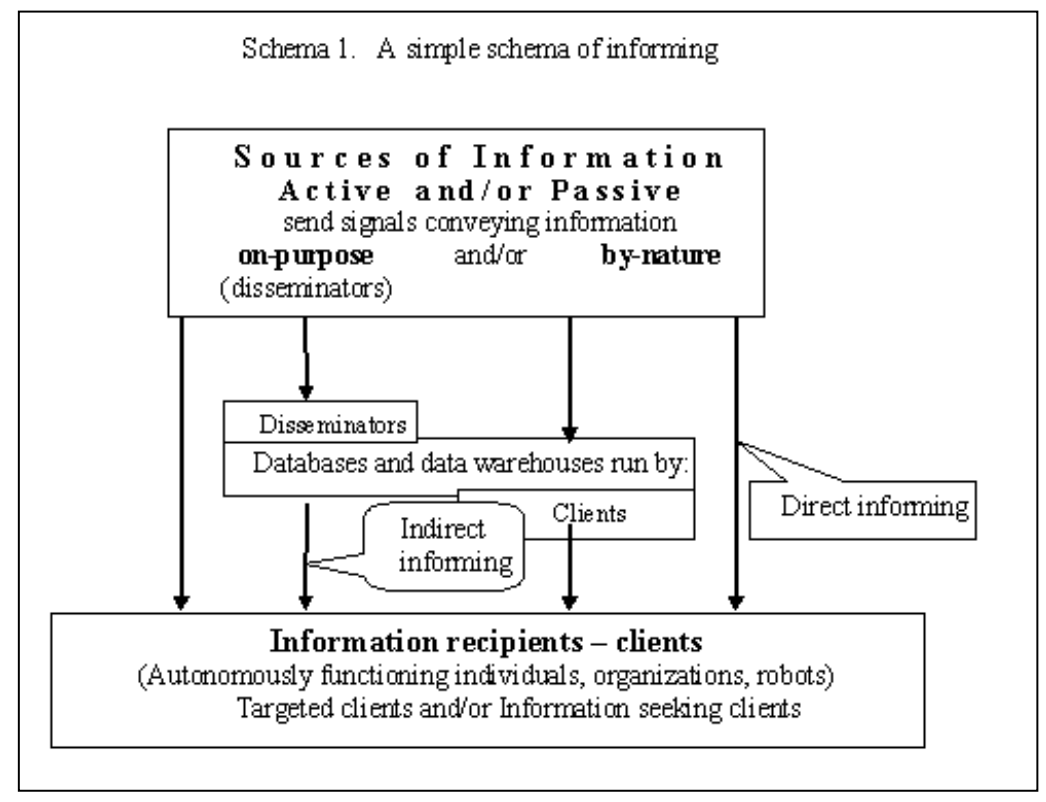

The design and examination of informing systems differs considerably for active and passive informing. In case of active informing, senders or disseminators try to provide gainfully some information service or to influence the behavior of the target. Usually, their perspective carries more weight than the clients' perspective, for disseminators affect the behavior of many clients. Tudjman \& Mikelic (2003) emphasized the exami-

nation of informing also from the sender's viewpoint. In passive informing, the information receiving entities may purposefully try to gather intelligence about the environment or obtain some feedback about the state of the situation under consideration if the results of their actions differ or may differ significantly when acting without the obtained information. In either situation, the senders (in active informing) and clients (in passive informing) expect measurable or at least perceivable results of some utility value or payoff. Hence, in cost-aware business environments, when one aims at achieving the best results, one should carefully examine the expected cost effectiveness of the respective informing systems. Schema 1 summarizes visually this way of viewing informing.

\section{The Purpose-Focused Framework for Examining Informing}

The purpose-focused framework (Gackowski, 2004a), selected for a rational inquiry into the problems of informing systems in business environments, takes the operations research (OR) approach and refers to the decision situations it serves. One assumes:

- A relatively complete qualitative cause/effect diagram, known also as a fishbone diagram, is available or can be drawn. It identifies the major factors affecting the expected business results, the business situation itself, and/or the required actions to implement the decisions made. Based on the data/information available, the end user makes pertinent deci- 
sions and takes subsequent actions. The results of the actions can be measured: by the sender - in active informing, the client - in passive informing, or by both.

- An analysis can reveal the relative strength of each factor by its impact on the main business purpose. In business, various criteria are used to measure the main purpose of its operations such as net income after taxes, retained earnings, return on investment, return on equity, etc.

- One can develop an informational model of the decision situation under consideration by taking inventory of what is already known, and what still must be acquired. That what is known, given, or available constitutes the data component of the model. Anything that is not yet available, but must be acquired by proper intelligence or delivered (sent, disseminated, and broadcasted) constitutes the informational component of the model.

The data component of the model is here the set of variable values that symbolically represent what is already known (objects, events, and their states) about the situation. The informational component is here the set of variable values that symbolically represent what is not yet known (objects, events, and their states) about the situation and must be acquired, gathered, measured, counted, etc. Those information values may change the business results, and/or the decision situation by itself, and/or the actions to implement the decisions made. Such a framework enables a rational examination of informing systems, and their cost effectiveness.

The main function of any informing process is to move the necessary information from its source to its target. The main purpose of any informing process is to contribute to the main business purpose of the organization it serves. Those contributions or payoffs by Marschak \& Miasawa (1968) are functions of the informing systems deployed. In economically sound environments, informing systems should be cost effective. When the generated effects are not worth sufficiently more than the cost incurred by the corresponding informing systems, there is no sound business reason for such projects.

The criteria of what constitutes an acceptable level of cost effectiveness must be determined within the context of the business environment the project serves. In addition, there are always other requirements and constraints imposed upon any project. The above should be articulated in formal project requests or requests for proposal for the respective informing processes.

\section{Project Requests or Requests for Proposals (RFP)}

The responsibility for the articulation of such documents rests with clients for passive informing and with disseminators for active informing. Many of them, however, succumb to the myth that project requests are of too technical a nature. It is exactly the opposite. The purpose of an RFP is to communicate to developers the will of the paying client or disseminator about the business purpose of the project within a framework of requirements, constraints, and evaluation criteria. It should not be technical at all; it should be business-like. The developers of informing systems and the IT professionals are responsible for finding and offering technical solutions.

Information system development is a separate branch of system engineering, but not so different that it cannot benefit from the accumulated experiences of its other branches such as industrial or manufacturing engineering. The presented approach to the preparation of a good project request was inspired by the IDEALS concept suggested by G. Nadler (1970) for work design and later adopted for IT applications by Gackowski (1991). The guiding concept of this approach is to communicate the business purpose of the project without overly constraining the developers in their search for solutions. 
Good project requests should specify: (1) Client or disseminator organizations' main business objectives expressed in terms of observable, better yet, measurable business effects to be attained for the common purpose. (2) Requirements and constraints, if possible, only those externally imposed upon the project and the informing process. (3) Time and spatial boundaries of the informing system and the project. (4) Evaluation guidelines of the project.

\section{Main business objectives}

Main business objectives (of the served organization) are defined as only those effects that separately and independently of each other constitute a sufficient reason for designing, building, and operating the target system that serves its main purpose, for instance increase in net profit after taxes. Usually they are considered critical and vital for the organization at the current state of business affairs such as winning a certain segment of consumers for products, in the case of active informing, or gathering information about additional economic indicators that substantially affect business results, in the case of passive informing. They are the critical success factors.

A frequently encountered weakness is lack of clear distinction between the main outputs of systems and their main purpose. For instance, the AIMQ methodology (Lee et al., 2002) focuses the examiners' attention on information products. It is not merely a terminological purism. It profoundly affects the way developers think about their task, where the real business problems to be addressed are, and how the informing process should be designed to support the attainment of the business main objectives, which in a tightly run business organization should contribute to its common purpose. Most project requests are usually overloaded with technical aspects, but short on stating business objectives to be attained.

Some reviewers are concerned with the situation of a composite business entity consisting of several entities with different purposes. This is exactly what one should not tolerate. Eventually it leads to the system's disintegration. All sub-entities must serve exactly the same purpose, for instance profit, but they may contribute to the common purpose by pursuing different objectives that depends on the nature of the subunit. For instance, on one hand, profit centers such as R\&D by developing a new highly profitable product line, marketing by aggressive advertising, service centers by attracting a new segment of customers, etc. On the other hand, cost centers may contribute to the same main purpose of corporate profit in a different way; manufacturing by cutting product manufacturing unit cost, inventory control by reducing inventory related cost per sales dollar, accounting by computerizing their operations and cutting cost per posting, etc.

\section{Requirements and constraints}

The fact that business problems must be solved and information systems have to function within a specific organizational environment results in many requirements and constraints imposed upon the project. They may pertain to any topic or aspect listed in the work system specification matrix (Gackowski, 1991). Those requirements and constraints should be divided into mandatory and optional ones. Mandatory means that the project will be abandoned or rejected if any one of those requirements could not be met. Optional or non-mandatory means that each one should be met only if meeting such requirements adds value to the project.

In order to facilitate design decisions with respect to meeting the optional requirements and constraints, the project request must contain a utility value associated with each of them. The value can be expressed in terms of expected additional benefits, acceptable higher costs, or acceptable trade-offs with other system parameters. For instance, in informing systems a requirement of fullfledged comments and explanations in natural language, on one hand may impair the delivery system's response time, while shorter and less user-friendly encoded messages will require 
trained users. In marketing, however, the same "full-fledged comments" may shorten not only the clients' response time, but also increase the response ratio, which is of utmost importance in marketing efforts. There are many such trade-offs possible. It is a complex issue. Meeting the optional requirements affects in different directions the gross business results and the cost of system's implementation and operations.

\section{Boundaries}

One has to consider the time and spatial boundaries of projects. Clients or disseminators should set the desired time boundaries in the most flexible manner, for instance the earliest date of a window of opportunity (from the viewpoint of the affected business) when it may be implemented. Inevitably, there are trade-offs in quality between a fast, simple, and dirty solution and a more carefully designed process. It is likely that the former will not be cost-effective over the long term. If circumstances permit, one should leave some flexibility and consider a range of alternatives. The project team should not be pushed towards a quick and dirty approach. However, the sooner the project is implemented the sooner the benefits begin to accrue, which favors rapid development. Nevertheless, it should not be done at the expense of quality.

Omission in defining the spatial or data flow boundaries of target information systems (a frequent error) can also lead to misunderstanding regarding their scope and mode of interfacing with other information systems and the embedding organizations. It may lead to intolerable discrepancies between what really was intended and what the team will propose, even when using the same evaluation criteria. Definition of time and spatial boundaries should be of equal concern to information disseminators and information clients. The case, when incremental design and deployment is planned, does not diminish the importance of this issue. It only requires additional functional decomposition of the system into application units with their own boundaries.

\section{Evaluation Guidelines}

Usually, the effects of informing are multidimensional. Disseminators or clients cannot evaluate the payoffs or benefits of attaining the business's main objectives or the benefits of meeting different requirements unless they can be expressed in a common scalar measure. In systems engineering, conversion to common units is attempted by various forms of utility or goal functions. The value of such a utility function becomes a valid evaluation criterion of all benefits only if it faithfully reflects the disseminators or clients' preferences with regard to:

1. Conjunctive fulfillment of the entire set of mandatory requirements and constraints

2. The main business objectives and the degree to which they are attained

3. Additive fulfillment of all the optional requirements and constraints imposed upon the project and the target information system.

Such evaluation criteria constitute a precondition for a full evaluation of the cost effectiveness of informing processes. The first component is a non-negotiable mandatory condition; otherwise, the project has to be aborted. With regard to the second component, not all the main business objectives must be attained, for by the definition of main objectives, meeting even a single one makes the project viable. A problem may arise when the evaluations of the progress on different main business objectives cannot be reduced to single common scalar value the same way as prices on a free market help us to express the utility value of different goods in the form of their replacement cost or exchange ratios expressed in the same monetary units. Similar problems may arise with regard to the valuation of the benefits related to meeting optional requirements and constraints. In 
a business setting, the evaluation guidelines should explain how to translate the meeting each optional requirement into additional benefits or cost reductions.

Considerable effort should be made to arrive at such a common agreed valuation; otherwise, rational decisions on potential trade-offs will be compromised. Neither disseminators, nor clients nor experts can objectively evaluate the cost effectiveness of the informing process when project requests do not provide relevant evaluation guidelines. Later, when the process is operational, no one can objectively determine whether it is run as effectively as projected.

In summary, a good project request should make it clear to the project team what is expected with regard to all the aspects mentioned. It should clearly communicate the way the recommended solutions and the developers will be evaluated, and how the solution will be compared with other alternatives and projects.

\section{General and Specific Quality Requirements}

For an informing process to be cost effective the signals and the information carried by them must meet many requirements with respect to many attributes of data/information quality. A brief description and discussion of the above follows according to their hierarchical, result-oriented taxonomy (Gackowski, 2004a).

1. One can subdivide the universe of all quality attributes into direct attributes and indirect or subordinate attributes. Changes to the values of direct attributes affect the results of business operations, while values of indirect attributes, as the name suggests indirectly affect the results, for they only determine or contribute to the direct attributes.

2. The direct attributes can be further subdivided into primary and secondary attributes. Changes of the values of primary attributes result in qualitative changes to the decision situations under consideration, while changes to values of secondary attributes quantitatively change business results. The latter are mostly of economic nature. In business, they become mandatory too, otherwise the use of data/information that does not meet the economic requirements would not make business sense.

3. Within the primary attributes, one must distinguish those pertaining to all values versus the situation specific ones. Some of them such as credibility and completeness are rarelyto-never attainable; they can be only desirable. Therefore, there is a pragmatic need to impose on them a mandatory requirement of being at least actionably credible, and taskeffectively actionably complete, otherwise there is no point in their examination.

The purpose-focused framework (Gackowski, 2004a) of data/information quality assessment makes possible their examination in an economical sequence.

\section{Direct Primary Quality Requirements in All Situations}

(They apply to each data/information value in all tasks are mandatory, and must be met unconditionally. Changes to the values of the respective quality attribute result in qualitative changes in the decision situations under consideration)

\section{(1) Interpretable during acquisition}

Messages or composite data/information may consist of one or more values. For them to be later usable at all, they must be interpretable within the process of their acquisition. In practice, interpretability means whether the received information value matches any state with some attributed or associated meaning in the mind of the receiving individual, or any state that automatically triggers a designed sequence of state transitions in the receiving numerically controlled device. Most 
authors omit it as obvious. When for any reasons the targeted individual or the receiving device is unable to interpret a data or information value, it is lost and it must be excluded from further examination.

Interpretability is contextual; a more educated receiver, a conditioned one, a trained one, or a different receiving device may still be able to interpret it. The information carrying signals must be noticeable to senses or sensors, discernible, recognizable, or identifiable. Thus, the latter are indirect attributes. These considerations are of major concern for users and disseminators. The interpretability of information values during their acquisition should not be confused with their presentational interpretability for users/clients.

More complex conditions and circumstances must be considered by information disseminators. A plethora of factors here comes into play. Many of them of a very subtle psychological nature how to effectively and gainfully reach the targeted client by employing the many findings, skills, and tricks offered by the art of communications, marketing, advertising, etc, which intersect here. They are subject of studies how effectively attract others' attention and how to persuade them into desired actions.

(2) Of significant impact, relevance by (Gleim, 2004) for CPAs, and by (Wang \& Strong, 1996)

The content, subject, or meaning of individual data/information values or any combination thereof must make a significant impact on the situation under consideration. In a business environment, impact should be assessed by the scope of changes made in the business situation itself and/or in the results of business operations, and/or actions taken to implement the decisions made. If the impact is insignificant from the perspective of disseminators and/or clients, the remaining attributes of the information item are irrelevant, too. Significant impact lends importance to all other dimensions of quality. The definitions of relevance referred to in the subtitle are far too narrow from the operations research $(\mathrm{OR})$ view.

Impact, however, can be quantified or at least ranked either from the viewpoint of information disseminators or information users. One may ask how the payoff or added value depends on the use of a specific information value, whether its impact is significant enough to warrant consideration. In a more rigorous manner, one may say that in a specific situation an information value may be qualitatively relevant but quantitatively irrelevant when its impact is negligible. If so, one should cease further examination of its remaining quality attributes.

It may also happen that the size of the impact may depend on other factors such as type of its availability: whether restricted only to a specific the decision-maker, fully unrestricted or anything in between. Restricted availability gives advantage to some decision makers over their competitors. Unrestricted availability of a data/information value may reduce that advantage to insignificance. These considerations are situation specific. (See "actionably timely available".)

One must also be aware of a very frequent case that a data/information value of a zero payoff or added value may still be of significant impact on the ultimate outcome. This takes place when it is a required companion of another information value that is associated with a significant payoff or added value. For instance, an emergency calls for roadside assistance with a well-defined payoff, but it must be accompanied by information values about the location. Without the latter, such a call cannot be effectively handled on its own.

(3) Actionably timely available, timeliness by (Gleim, 2004), accessibility by (Wang \& Strong)

Once all data/information items of significant impact are identified, the next direct primary and mandatory quality dimension is "actionably timely available" that is before it loses its capacity to make a significant impact. It pertains to individual data or information values or any combination thereof. If the data are not on time to meet the need, why bother about other requirements? In 
ever-changing reality, time is of the essence. Even with all remaining requirements met perfectly, if timely availability cannot be assured, the impact of late data/information values may be null.

The "actionable timely availability" may also be viewed differently. For instance, whether the data or information value under consideration is available exclusively to a single interested individual. Here, one deals with two exremes: with restricted or unrestricted availability. From the viewpoint of logical interdependencies among DQ/IQ dimensions, one can see here an interesting case of circular interdependence between two quality dimensions. In order to consider at all the "actionable timely availability" of any data/information value, it must be of "significant impact". In competetive envirnoment, however, frequently a significant impact of data/information may depend on its restricted availability. The latter is an example of situationspecific requirements that are task-specific and do not pertain to all values.

(4) Actionably credible, believable by (Wang \& Strong, 1996), reliable by (Gleim, 2004)

Only messages declared of significant impact and actionably timely available, which implies they were interpretable during acquisition, and were understood when presented, users/clients should test them for credibility; whether they are true, whether they can be relied on. The adjective true means consistent with reality. While probing for veracity, users/clients face dramatic options: whether they received valid information, misinformation, or disinformation

- Valid information faithfully represents or reflects reality. To this end, it should be objective (unbiased), accurate (error free), precise, and current (up to date). Usually this is assumed, when information is of proven authorship, from a reputable source; can be replicated, confirmed otherwise, or traced back to the responsible originator, where the level of responsibility should be adequate to the potential consequences of possible errors.

- Misinformation unintentionally misrepresents reality. It may be distorted at its acquisition, communication, storing, processing, presentation and the interpretation by itself.

- Disinformation intentionally misinforms. On one hand, in simple cases, it may not be clear who the originator is due to omission of contact addresses, when it was originated or updated, what methods of collection or acquisition were used, etc. On the other hand, all the above is present and available. Now, however, the user or client faces two extremes of deception with many possibilities in between. All the above listed indicators of validity are given: (1) To appear legitimate, but one or more of them are false, or (2) Are true, and usually presented in a very motivational manner, but actually the intent of the message is deliberately malicious, criminal, aimed at trapping the gullible.

Credibility is a complex function of many indirect attributes of quality, in particular when computer and communication technology are widely used. One can summarize it as follows: The clients trust in or the credibility of the data/information presented to them hinges on: (1) Traceability that facilitates assessment of the sources' reputation. (2) Faithful mapping of the real-world states into states of the delivery system, and then later into the data/information values conveniently presented to users/clients. The reputation of data or information sources is a function of at least the following quality prerequisites (indirect attributes): objectivity (unbiased), accuracy (error free), precision, and currency (up-to-date). Deficiency in each of them contributes to loss of credibility. They also must be examined in their natural sequence.

Credibility can also be increased in other ways, for instance when the same data/information values can be acquired from a variety of sources or can be confirmed by other measurement methods or computed. Credibility may also be assured when the specialized business process of massive data/information acquisition, entry, verification, validation, storing, making them available, and converting them into presentation interpretable format is subject to stringent quality requirements, 
tests, and audit procedures. The more important, valuable, dangerous, litigation prone, and/or vulnerable to criminal activities a transaction item is, no responsible manager would neglect traceability and transparency of its handling.

Since credibility is rarely-to-never fully attainable, in many situations, users must learn to act with only an acceptable level of credibility labeled actionably credible. For practical purposes, actionably credible can be defined as the degree of credibility at which the user is willing to take action. The definition is precise, but the actionable level of credibility again is a function of the decision situation, including the personality of the decision maker in particular. It is scalable and should be examined only after a proper ranking and comparison of all task-specific factors of significant impact took place, while checking for task-effective actionable completeness of the set of task-specific data/information values.

Actionable credibility closes the list of the four data or information quality attributes that defines by enumeration the task-specific usability of single data/information values in all situations of direct informing when the same individual when there are no intermediaries between the source and the user/client. It is usable from the senders and/or targeted clients' viewpoint. Usability indicates that a data/information value may be used but not necessarily effectively.

In most organizations, however, due to division of labor data/information acquisition and their use are completely separated, thus indirect informing takes place. In such situations, users must be presented with data/information that is interpretable and understandable for them, for instance legible, in their preferred language, measurement units, conventions, etc. Then data/information must also be presentation interpretable and it becomes another mandatory quality requirement.

\section{Effectively task-specifically operationally complete}

Completeness of data/information pertains to a set of the identified task-specific factors of significant impact. Once one arrived at a task-specific set of usable data or information values, one can test their completeness with regard to the situation under consideration. Completeness of data or information values in decision-making, in contrast to their mapping while storing, processing and presenting them is more complex than it appears on its surface. It is strongly related to their impact as defined earlier. One must distinguish at least two types of completeness: operational completeness and congnitive completeness.

Within the context of decision situations, operational completeness measures the degree to which the data/information values of significant impact are available. Operational completeness may be measured in percentage points $(1-100 \%)$ as the ratio of the sum of all results that can be attributed to the corresponding values available and the sum of results attained. In real-life situations, usually, some residual operational results remain unaccountable. This means it is not possible to attribute them to any previously identified factors. They may be used as a relative or absolute measure how incomplete the impact analysis is. Figure 1 illustrates the general interdependence between impact and operational completeness of data/information items.

Murkier is the qualitative or cognitive aspect of completeness of data/information values in a situation under consideration. In real life situations, in the fight for survival, on a battlefield or in global business competition, one may never be certain whether all relevant success factors or dangers are identified and evaluated. Prudence requires gathering more information to inform interested decision makers so that they may assess all the maybe not yet perceivable but potentially possible critical factors for planning of counter measures and contingency provisions. The critical blow most frequently comes from a danger or direction not identified and recognized in time. It cannot be considered mandatory on its own merit, for it is rarely-to-never fully attainable. Figure 2 illustrates the general interdependence between impact of data/information values about 


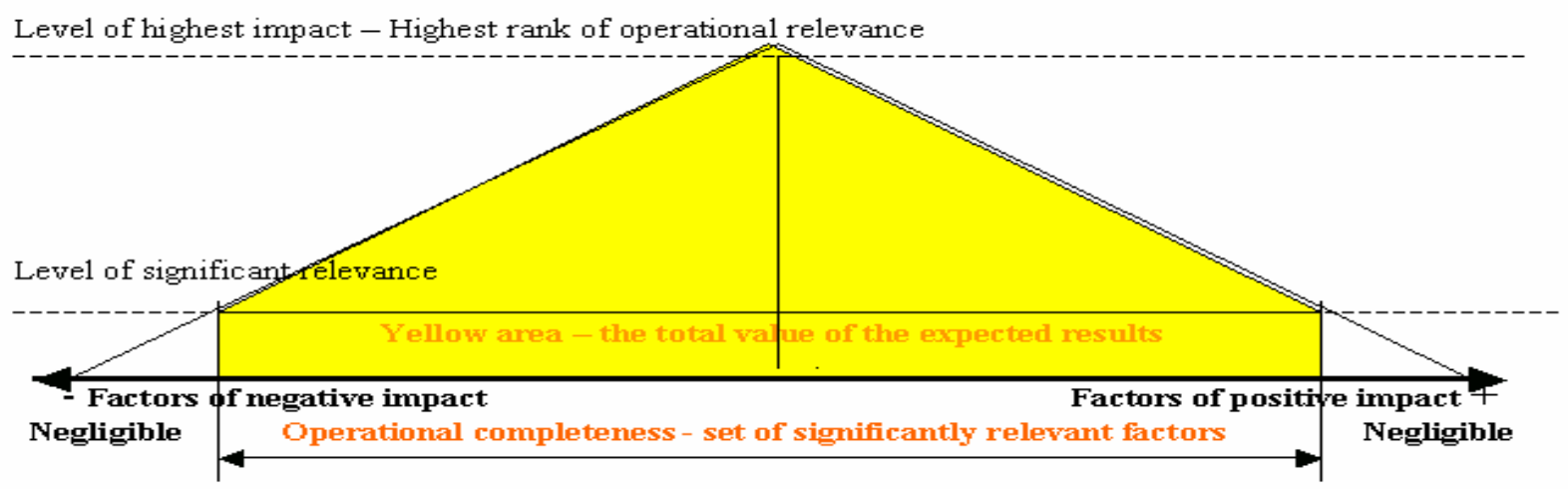

Figure 1 The relationship between significant relevance and operational completeness of data or information items pertaining to the corresponding factors that determine and contribute to the total value of the expected results in a decision situation under consideration.

all identified hypothetical factors pertaining to a decision situation under consideration and the fuzzy notion of their cognitive completeness.

Both Figures $(1,2)$ illustrate how the quantified impact of data/information values determines the operational and cognitive completeness of the totality of factors pertaining to a specific situation. Since both are rarely-to-never fully attainable, managers must frequently act based on an incomplete set of data/information values. Like credibility, completeness is measured by a continuum of degrees. From the purely pragmatic viewpoint, there is at least one important degree of completeness, when it becomes operationally complete. It is, however, not enough to act, one wants to act effectively. Therefore it is necessary to distinguish qualitatively at least four kinds of taskspecific operational completeness that determines the level of usefulness of any set data or information values. Gleim (2004) warns "Without usefulness no benefits are provided."

Level of highest impact - Highest rank of potential relevance

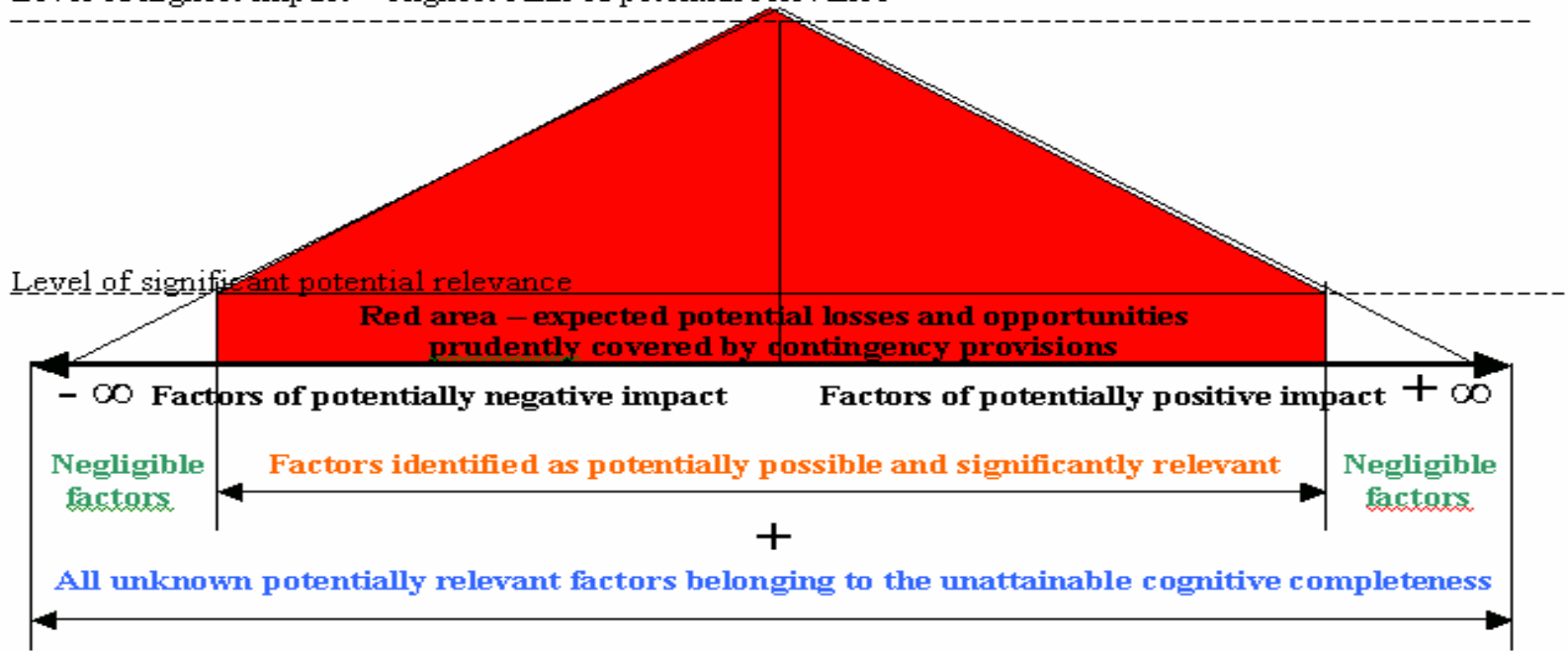

Figure 2 Relevance of data/information items about identifiable potential factors pertaining to a decision situation under consideration and the unattainable notion of their cognitive completeness.

When economy of operations is secondary to operational effectiveness, one may be satisfied with

- Effective task-specific operational completeness only, which is attained when within the set of task-specific usable data or information values, there is at least one value of significant payoff or added value. This is a situation of an all out effort when at least temporar- 
ily economy is of secondary concern. Then all the direct secondary requirements of data/information quality discussed later need not to be tested.

When, however, economy takes precedence over other considerations, all the later discussed direct secondary quality requirements become mandatory, as well. Than, one may distinguish three other levels of task-specific operational completeness:

- Economical task-specific operational completeness - attained when the sum of all payoffs or added values exceeds the sum of all costs of the operations under consideration.

- Cost effective task-specific operational completeness - attained when the ratio of the sum of all payoffs or added values divided by the sum of all operations costs exceeds the required level.

- Expected cost effective task-specific operational completeness - computed as above, but as a function o expected payoffs and expected costs.

Operational and cognitive completeness must be considered mainly by information users or clients; however, these should not be completely ignored by information disseminators. The latter, in their messages, they also ought to consider addressing the potentially possible concerns of clients. Thus, task-effective actionable completeness of a set of task-specific usable data/information values is the sixth direct primary mandatory quality requirement of general nature after actionably credible or believable.

This discussion closes the list of the five direct primary mandatory requirements of data or information quality, which pertain to all values and situations. Sometimes, there may be other additional situation specific quality requirements such as the before mentioned exclusive or restricted availability. What follows is a discussion of the direct but secondary attributes of data or information quality, which are of economic nature. In business, where cost effective projects are required, meeting all the direct secondary quality attributes makes them economically useful, and then, they are mandatory, too.

\section{Direct Secondary Quality Requirements (When Economy is an Issue)} (Changes in values of the respective data/information quality attribute result in quantitative changes in business results)

One may ask how sensitive a situation is to the use of any specific data/information value; whether it has a significant impact on results worthy of consideration. The quantified and ranked impact carries all the gross benefits or payoffs possible to attain and provides the examiner with a situational reference scale. It suggests how much attention one should pay to each data or information value relative to other with regard to the four other primary attributes of their quality. Hence, one must test the economic level of acquisition interpretability, actionable timely availability, actionable credibility, and their presentation interpretability.

Changes in these requirements are additive. Therefore, the sequence of their examination is formally irrelevant. Nevertheless, all items can be economically evaluated only after their proper ranking during a completeness check. In business environments, however, data/information values, separately or collectively in composite combinations of them, must also be economically useful. Therefore, in business, the direct secondary quality attributes are mandatory, too. For instance, when a single or a composite data/information value cannot be economically acquired, one should cease examining the remaining direct secondary attributes. The same reasoning applies to the remaining attributes. 


\section{(1e) Economically interpretable during acquisition}

There is no doubt that interpretability of incoming information values during their acquisition is the first direct primary and mandatory requirement that must be met in order to trigger the chain of further examinations. In certain situations, however, it may be attainable only at a prohibitively high cost in comparison to the associated payoffs that it is not worthy the effort. This cost may entail the cost of decoding, translation, maintaining a system of early detection and warning about dangers (tsunami, earthquake, missile attack, etc.). Therefore, the first question to ask is whether it is technologically possible at all. Only after all mandatory requirements are met one should ask whether those requirements can be met economically as it is required in business environments. In business environments, economy is mandatory. Only when interpretability of information value during their acquisition is economically attainable, it opens the door for examination of the remaining direct secondary requirements. On the other hand, when the stakes of national security, for instance, are higher than the established economical criteria, the concerned data or information values still remain effectively usable, however, not economically.

\section{(2e) Economically actionably timely available}

Meeting mandatory requirements usually does not add value; it only makes the data's value usable. In business, however, the cost of making a value timely available should not exceed the associated payoff. In addition, timely availability is scalable. One may receive the necessary data or information not only on time, but also more or less in advance. The cost of rendering them sooner "actionably available" should also not exceed the associated additional benefits. The additional time may be used for making decisions with less haste, and/or for better preparation of actions. Hence, one may also obtain better results when additional time is available. Providing additional time may add value, but it may cost more. On the other hand, excessive additional time can cause a deterioration of business results due to human forgetfulness or possible distractions between the time of early warning and time for action. There is no analytical formula to estimate it; however, in specific situations it may be possible to determine the best timing experimentally. Whether it is worth depends on the difference in results it makes and on how much it will cost to accelerate the informing process or increase its frequency.

\section{(3e) Economically actionably credible}

Actionable credibility may be compromised by deficiencies in several other indirect quality attributes for instance by lack of objectivity, accuracy, precision and currency.

Loss of objectivity meant as free from bias may happen in the process of data/information acquisition process due to the approaches and methods used in selecting the primary sources, measuring points, observation points, and finally, when collecting, processing and presenting data. The resulting bias may be either unintended due to ignorance or introduced intentionally. In both cases, the results of such distortions may be significant, and in the latter case, deceptive and damaging. To rectify the bias and compensate for it may require engagement of substantial additional resources. Whether it is economically justified, it can be estimated only when the size of its impact on the results is significant enough.

Another problem is accuracy meant as free from errors, among them random errors. One encounters them in all situations. Usually accuracy is indicated indirectly by inaccuracy of data or information values, which is the complement to one of accuracy. Inaccuracy or error rate equals one - accuracy. A typical gross measure of inaccuracy in this sense is the error rate. One calculates it by dividing the number of values in error by the total number of data or information values gathered. In practice, a more useful measure of inaccuracy due to different kind of errors is the expected cost of dealing with their consequences. One may calculate it by multiplying the number of data/information values by the probability or frequency of each type of error by the 
average cost of dealing with each type of them. This measure of inaccuracy provides the clients with a better idea how serious the consequences of each type of error are. One may reduce many of them by using check digits, error self-detection codes, error self-correcting codes, etc. A good example is the use of barcode readers, which considerably reduce many errors. Clients of information systems, even business systems analysts, need not to be experts in dealing with such situations, but they should be taught to recognize the need for those measures.

Finally, one may encounter low precision in the representation of the reality. For numerical data, precision is measured by the number of significant digits used. One measures the precision of pictures and images by the number of dots per inch. This unit is commonly used to describe the precision of printers, computer screens, scanners, etc. Insufficient precision of data/information presentation may compromise the results obtained

There is a trap associated with accuracy and precision. Generally, they are overrated (Wang, et al, 1996). Unchecked efforts to increase the level of accuracy and/or precision of any data or information value can become counterproductive. The ultimate determination of the indispensable and economically justified level of any of them strongly depends on its utility value. For more details on how the level of accuracy or precision or data/information determines its utility value and procurement cost, hence cost effectiveness, see Gackowski (2004a).

Currency of data/information values means here whether it is adequately up to date. It was labeled timeliness by Wang \& Strong (1996) and defined as "The extent the age of the data is appropriate for the task at hand." The label timeliness is used there in conflict with the terminology used by CPAs for timely availability causing unnecessary confusion. The frequency of updates should be optimized, where possible, for either insufficient frequency or too frequent updates are detrimental to the cost effectiveness of the informing delivery system.

Maximum business benefits from using data/information values can be attained only at their optimum level of objectivity, accuracy, precision, currency. Finding this optimum is not easy, but the truth is that it lies somewhere between the low and high level. For instance, whenever information technology professionals tempt clients with higher accuracy, precision or currency than they had before, they should ask bluntly "What will be the additional business benefits and at what additional cost?" When one has no indication that their increased level leads to higher cost effectiveness, forget it. To the surprise of many, one thing is sure; the examination of their economic level should be postponed nearly until the very end...

(4e) Economically presentation interpretable or conveniently presented

Ease and comfort, in educational services or computer games, even fun, of use of data or information is related to its form, format, and mode of delivery. It may affect how fast end-users read, perceive, interpret, comprehend, analyze, absorb, draw conclusions, react, and finally act upon it. Within this category, one considers clarity, consistency, order, media used, level of summarization, user-preferred type of presentation such as text, graph, diagram, picture, esthetics, etc. In case of composite information, these properties are determined not only by its components but also by the way, the components are combined. Some deficiencies in this respect rarely preclude the use of the affected data/information values. They may increase or decrease the convenience of their use and their procurement cost, hence subsequently their expected cost effectiveness. The outcome of examining the economic level of the previous four indirect conditions of data/information credibility determines whether it is economically attainable.

When combined and summarized, all the direct secondary quality attributes determine the economic usability of the data or information concerned, and ultimately, when combined, the cost effectiveness of the delivery system. The same aspects, when considered from the viewpoint of information disseminators carry more weight. Even subtle differences in these aspects may decide 
whether clients respond at all, and how they respond. One must also avoid a one-sided assessment of the cost effectiveness of informing that is only from the disseminators or only from the clients' viewpoint. In an ideal solution, it should be cost effective for both sides. This is what makes a business relationship lasting and successful.

\section{Usefulness and Cost Effectiveness of Informing}

There is a need for a distinction of usability and usefulness of data or information. First, data or information values or any combination thereof should not only be usable but also useful. In operations, it means they can be used effectively. To this end they must be: interpretable during acquisition, of significant impact, actionably timely available, actionably credible, (if situation requires, interpretable during presentation), and belong to effectively task-specifically operationally complete sets of such values.

There may also be other important situation specific requirements for effective usability of information, for instance exclusivity or restricted availability. Usually, effectively usable data or information may also be useful, when the ultimate goal is to attain the desired purpose by whatever it takes in an all-out effort regardless of the cost. Such attitudes dominate in warfare for ideological or religious reasons. Usefulness, however, is contextual, depending heavily on the situation.

1. For general education purposes, any message (which may consist of many data values) is useful that broadens students' perception of the world, society, and community.

2. For designers of decision support systems any data/information is useful that changes the outcome of decision situations under consideration.

3. For business organizations, any data/information is useful economically or cost effectively with regard to the results of business operations, and/or the business situation itself, and/or at least the actions that implement the decisions made.

Whether a task-specific set of usable data/information values is cost effectively useful depends on whether it is effectively operationally complete and meets all the economic requirements of data or information quality such as: economic level of interpretability during acquisition, actionable timely availability, (if necessary, presentation interpretability), and actionable credibility in such a degree that the ratio of the sum of all payoffs or added values divided by the sum of all operations costs exceeds the required level.. In business environments, all the economical quality requirements are mandatory, too. Economic or cost effective usefulness of task-specific sets of data or information values should be of foremost interest to informing clients and disseminators.

Rarely, however, does anything useful come at no cost and at no risk hence, the real cumulative measure of usefulness should be the expected cost effectiveness of the entire informing process assessed from the viewpoint of the purpose of the business entity it serves. It can be evaluated by either objective criteria, when an adequate model of the decision situation exists or post-facto, after deployment of the informing system.

This explanation is necessary, for in some papers on informing science the term usability is given a very different meaning such as (Tudjman, 2003): "Usability as a measure of efficiency of the information process ..." One can easily see that for data or information values to be effectively usable they must meet at least the five direct primary mandatory requirements of general nature. His assertion that "Relevance is an accepted term for measuring information usability by the user" is not true. Usability is not synonymous with relevance, or better "of significant impact." It is only one out of many mandatory necessary requirements for data or information values to be effectively usable. Irrelevant data or information may add cost and decrease usefulness. Even 
relevant values may not be effectively usable, when they are not actionably timely available, presentation interpretable, actionably credible, or are not members of effective sets that are taskspecifically operationally complete.

Before the expected cost effectiveness can be discussed, one must define the utility value and procurement cost of data or information, their net utility value, and their simple cost effectiveness. These issues, however, were presented in (Gackowski, 2004b)

\section{The Informing Science Framework - Suggested Refinements}

In 1999, Eli Cohen defined the Informing Science Framework. As a byproduct of this rational and technology-independent inquiry into informing systems, a need emerges for some refinements of the framework with regard to information - not the quantity of information, but its utilitarian usefulness in decision-making, and with regard to the informing delivery system.

Within this framework, Cohen refers to Shannon's model, where information is defined as reduction in uncertainty, or reduced entropy experienced by the receiver. That which has been mathematically defined by Shannon is the amount of information or the quantity of information the reduction of entropy of the system. It is not a conceptual (or a "real" by Leibniz) definition of information in its essence. "We should not confuse a measure of a thing with the thing measured, let alone to confuse the metric with the thing measured by" as neatly explained N. Callaos \& B. Callaos (2002). The reduction of entropy pertains to a closed system, as is the case with communications systems. Based on this premise, Cohen says, “... information is defined in terms of the receiver's level of uncertainty. In the field of Information Systems, we would say information is defined as that which reduces risk for the decision-maker." This, however, only may be so.

Business organizations, by their very definition, are open systems; they are work systems or operational systems that exchange inputs and outputs with their environments. From the perspective of decision science, a new information value may actually increase the level of uncertainty or the risk level, for instance due to information about new threats. Similarly, the entropy associated with such decision situations increases. (It can be precisely computed, when an adequate model of the decision situation exists.) This does not happen in a closed system. Thus within the confines of defined decision situation models, one may say: any received value is information that from the client's viewpoint is (1) new or unknown, and/or (2) changes any existing variable value about the situation under consideration. It may change the business results, and/or the decision situation by itself, and/or the actions to implement the decisions made.

Information is defined here in a pragmatic manner within the confines of decision situations. It seems that information is task-specific usable, when it meets at least the five direct primary mandatory quality requirements of general nature: interpretable during acquisition, of significant impact, actionably timely available, presentation interpretable, and actionably credible, though in special situations, more attributes must be considered. Significant means here that the changes exceed a predefined threshold level that warrants the clients' attention. Task-specific usable information may be useful, but not necessarily economically useful.

Pragmatically oriented business people, however, require more. They need data/information that is useful. A single data/information value, however, can never be used on its own in isolation; it is useful only when it is an indispensable member of an effective task specific operationally complete set of task specific usable data or information values. Such a set must have at least one value of significant payoff or added value. The ultimate goal of examining data/information values for the above discussed quality requirements is to arrive at such sets. When economy of operations is of primary concern, on should distinguish at least three other levels of task-specific operational completeness of data/information values: economical, cost effective, and expected 
cost effective, as it was explained under operational completeness. Therefore in business environments, the direct secondary dimensions that are of economic nature are mandatory, as well.

Part of informing is done by delivery systems, which are one of the three main components of the informing science framework. Here, the description of delivery systems needs some expansion. It is true that "Information technologies are not limited to computing, they entail also other media that augment communication such as video, voice, and personal contacts." However, "impersonal contacts" should be added, too. They occur in small-scale applications. Simple arrangements of physical objects (stocked merchandise) can create effective informing systems (Gackowski, 1982). Small inventory control applications for street-corner shops commonly used this approach in the pre-computing era. Such informing is purely "impersonal." In small-scale applications, it still may the simplest and least expensive solution, therefore hated by computer manufacturers.

Again, some reviewers ask why usefulness is as closely associated with changes as defined above. They use an example of information that "helps" to support a preliminary decision rather than change it. The definition of changes given above does not even mention the change of decisions made; however, it may imply this. The answer is perhaps counter-intuitive but simple. One must be aware that any reduction in risk or uncertainty is a real change in the business situation under consideration. Its entropy decreases. In an extreme case, incoming information may reduce uncertainty practically to zero, or increase the decision maker's certainty to one. In decision science, it means that the previous stochastic model of the decision situation is reduced to a deterministic model. This is a serious qualitative change by any measure. Subsequently, it requires different mathematical methods to solve the problem.

Thus, there are several conclusions for this section that might be considered. The Informing Science Framework (Cohen, 1999) might be extended by the following changes:

- "In the field of Information Systems that support management in attaining their goals, information is defined as values of symbolic representations of reality (objects, events, and their states) that change the business results, and/or changes the decision situation by itself, and/or changes the actions necessary to implement the decisions made. Pragmatically any information value must first be usable. It becomes usable by meeting a defined set of mandatory task-specific quality requirements. But it may become useful only within an effective set of data/information values that is task-specifically operationally complete. When economy does not matter, usable information may also become useful but not necessarily economically useful. In business it must be economically or cost effectively useful by meeting an additional defined set of secondary quality requirements." (This addition points out the many quality requirements that prevent informing from becoming information pollution.)

- "Information technologies are not limited to computing. Communication includes video, voice, and even personal and impersonal contacts, when it is planned."

Even information that confirms an uncertain assumption does change the decision situation. Of course, it must be of significant impact that warrants the decision-makers' attention.

\section{Conclusions}

One may conclude the rational technology-independent inquiry into informing systems viewed from the teleological purpose-focused perspective with the following observations:

- Examination of informing processes requires a clear distinction between active and passive informing for their examination, design, and operations differ considerably. 
- In active informing the source is active, it becomes the sender or disseminator of the information and tries purposefully to change the behavior of the target.

- In passive informing the source is passive, but the client tries purposfully to gather intelligence about the envirnoment or the state of of the situation under consideration, expecting that the payoffs of their actions differ significantly, when acting without it.

- Clients are always acting agents, therefore they must be relatively autonomous in their actions, whether individuals, their organizations, or devices (if devices, usually numerically controlled ones), to be able to act differently in response to received information.

- Clients can be active or passive with regard to the information flow, for they may actively search for the desired information or may more or less attentively wait for it, hence the information flow may be a solicited or unsolicited one.

- Measurable or perceivable results or benefits are expected; in active informing - mainly by the sender, and in passive informing - mainly by the client, but usually by both.

In business environments, the purpose-focused framework (Gackowski, 2004a) seems to serve best within the context of informing systems. Table 1 summarizes all types of data/information quality requirements for informing according to the taxonomy proposed by Gackowski in (2004a). As a byproduct of this inquiry, in a separate section, two suggestions with a rationale are presented on how the Informing Science Framework (Cohen, 1999) might be refined.

\begin{tabular}{|c|c|c|c|c|c|c|}
\hline & \multicolumn{3}{|c|}{ Examples of attributes of data/information quality } \\
\hline & & & & & Direct Attributes & Examples of Indirect Attributes \\
\hline \multirow{10}{*}{ 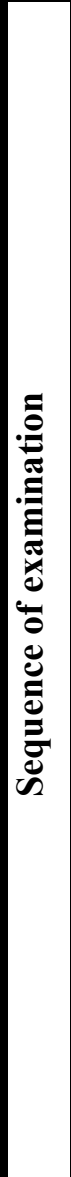 } & \multirow{6}{*}{$\|$} & \multirow{6}{*}{ 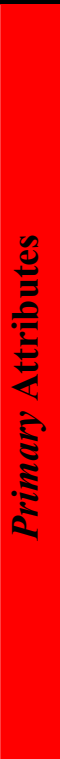 } & \multirow{6}{*}{$\begin{array}{c}\text { Mandatory } \\
\text { attributes } \\
\text { of general } \\
\text { nature } \\
\text { (Versus } \\
\text { situation } \\
\text { specific) }\end{array}$} & \multicolumn{2}{|c|}{$\begin{array}{l}\text { Acquisition interpret- } \\
\text { able }\end{array}$} & Discernible, recognizable, identifiable \\
\hline & & & & \multicolumn{2}{|c|}{$\begin{array}{l}\text { Of significant impact } \\
\text { or relevance }\end{array}$} & $\begin{array}{l}\text { Adds value, mandatory companion, } \\
\text { admissible, restricted access }\end{array}$ \\
\hline & & & & \multicolumn{2}{|c|}{$\begin{array}{l}\text { Actionably timely } \\
\text { available }\end{array}$} & $\begin{array}{l}\text { Mode of decision making (individual, } \\
\text { collective) }\end{array}$ \\
\hline & & & & \multicolumn{2}{|c|}{$\begin{array}{l}\text { (Presentation inter- } \\
\text { pretable) }\end{array}$} & Legible, understandable, \\
\hline & & & & \multicolumn{2}{|c|}{ Actionably credible } & $\begin{array}{l}\text { Disinformation, misinformation, valid; } \\
\text { traceable, faithfully mapped }\end{array}$ \\
\hline & & & & \multicolumn{2}{|c|}{$\begin{array}{l}\text { Effectively task- } \\
\text { specifically operation- } \\
\text { ally complete set }\end{array}$} & $\begin{array}{l}\text { Decision maker's traits: risk averse, } \\
\text { cautious, prudent, motivated, jumpy }\end{array}$ \\
\hline & \multirow{4}{*}{ 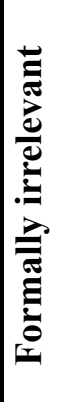 } & \multirow{4}{*}{ 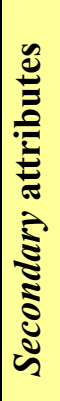 } & & \multirow{4}{*}{ 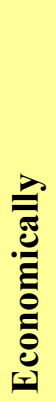 } & $\begin{array}{l}\text { Acquisition inter- } \\
\text { pretable }\end{array}$ & Requires decoding, translation \\
\hline & & & & & Timely available & How much in advance \\
\hline & & & & & Actionably credible & Objective, accurate, precise, current \\
\hline & & & & & $\begin{array}{l}\text { (Presentation in- } \\
\text { terpretable) }\end{array}$ & $\begin{array}{l}\text { Summarized, detail, text, graph, dia- } \\
\text { gram, picture, media, clarity, order, } \\
\text { consistency, mode of delivery }\end{array}$ \\
\hline
\end{tabular}




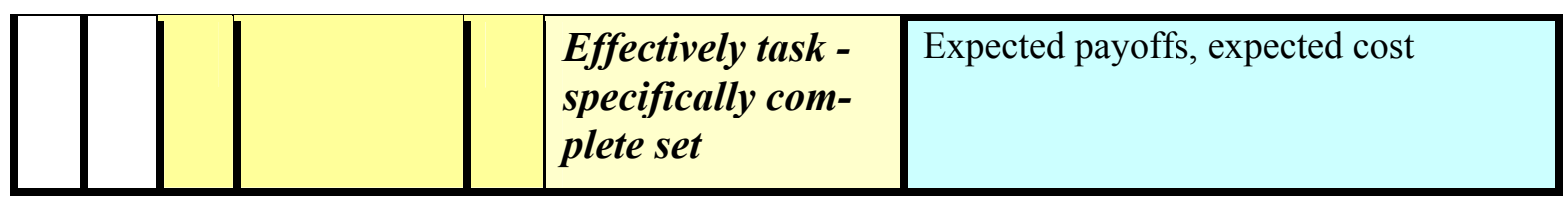

Table 1 Summary of data/information quality requirements in informing within the hierarchical result-oriented taxonomy in economic sequence of their examination

\section{References}

Alter, S. (2002). Information systems - Foundation of e-business. Prentice Hall.

Callaos, N., \& Callaos, B. (2002). Toward a systemic notion of information: Practical consequences. Informing Science Journal, 5, (1). Retrieved from http://inform.nu/Articles/Vol5/v5n1p001-011.pdf

Cohen, E. (1999). Reconceptualizing information systems as a field of the transdiscipline informing science: From ugly duckling to swan. Journal of Computing and Information Technology, 7 (3), 213-219. Retrieved from http://inform.nu/WhatsIS.htm

Dock, V. T., \& Wetherbe, J. C. (1988). Computer information systems for business. West Publishing.

Gackowski, Z. J. (1982). An approach to categorization of information systems. Proceedings of the ASIS (American Society for Information Science) Annual Meeting, Volume 19, Columbus, OH.

Gackowski, Z. J. (1991). System project request favoring designers' freedom. Proceedings of the Twentieth Annual Meeting of the Western Decision Sciences Institute, Lihue, Kauai, HI.

Gackowski, Z. J. (2004a). Logical interdependence of data/information quality dimensions - A Purposefocused View on IQ. Proceedings of the 9th International Conference on Information Quality - ICIQ 2004 at Massachusetts Institute of Technology (MIT), Cambridge, MA. Retrieved from http://www.iqconference.org/Documents/IQ Conference 2004/Papers/LogicalInterdependence.pdf

Gackowski, Z. J. (2004b). What to teach business students in MIS courses about data \& information. Issues in Informing Science and Information Technology, 1. Santa Rosa, CA: The Informing Science Institute. Retrieved from http://articles.iisit.org/110gacko.pdf

Gleim, I. N. (2004, $12^{\text {th }}$ edition). CPA Review Financial. Gleim Publications Inc

Huang, K., Lee, Y. W., \& Wang, R. Y. (1999). Quality information and knowledge. Prentice Hall PTR, NJ, (Quality Programs \& Initiatives at MIT - MITIQ Program).

Lee, Y., Strong, D., Kahn, B., \& Wang, R. (2002). AIMQ: A methodology for information quality assessment. Information \& Management, 40 (2), 133-146. Retrieved from http://mitiq.mit.edu/Documents/Publications/TDQMpub/2002/AIMQ.pdf

Malaga, R. A. (2005). Information systems technology. Pearson Prentice Hall

Marschak, J., \& Miasawa, K. (1968). Economic comparability of information systems. International Economic Review, 9 (2), 137-174.

Nadler, G. (1970). Work systems design: The IDEAL concept. Homewood, IL: Richard D. Irwin.

Nauman, F., and Roth, M., "Information Quality: How Good Are Off-The-Shelf DBMS?" Proceedings of the 9th International Conference on Information Quality (ICIQ-04), Cambridge, MA. Retrieved from http://www.iqconference.org/Documents/IQ\%20Conference\%202004/Papers/InfoQualityHowGoodar eOfftheShelfDBMS.pdf

O’Brien, J. A. (2003). Introduction to Information Systems. (Eleventh Edition), Mc Graw-Hill/Irwin

O’Brien, J. A. (2004). Management information systems (Sixth Edition). Mc Graw-Hill/Irwin

Tudjman, M. \& Mikelic, N. (2003). Information science: Science about information, misinformation and disinformation. Informing Science + IT Education Conference Proceedings (pp. 1513-1527), INSITE 
2003, Pori, Finland. Retrieved from

http://proceedings.informingscience.org/IS2003Proceedings/docs/204Tudjm.pdf

Wand, Y. \& Wang R. Y. (1996). Anchoring data quality dimensions in ontological foundations. Communications of the ACM, 39 (11), 86-95. Retrieved from

https://www.crg2.com/iqconference/documents/publications/TDQMpub/WandWangCACMNov96.pdf

Wang, R. Y., \& Strong, D. M. (1996). Beyond accuracy: What data quality means to data consumers. Journal of Management Information Systems (JMIS), 12 (4), 5-34. Retrieved from http://jmis.bentley.edu/articles/v12 n4 p5/index.html

\section{Biography}

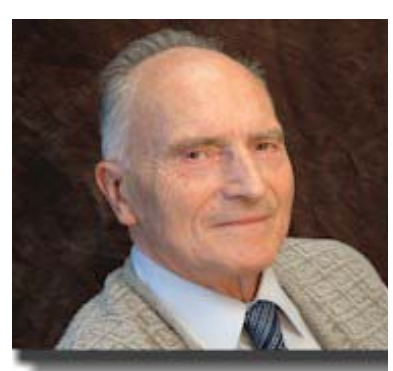

Zbigniew J Gackowski has extensive experience in industry, public administration, and universities. His teaching and research bridge the gap between Central European and US experience in Computer Information Systems [Warsaw Polytechnic, The University of Michigan (Fulbright Research Scholarship), Purdue University (Visiting Associate Professor), Baruch College (Visiting Professor), CSU, Stanislaus (Professor), The University of Melbourne (Visiting Professor].

His research has received much recognition. While in Poland, he published over 120 items, among them: 4 books, and 6 papers in refereed journals, 12 papers presented across Europe, United States, Middle East, and South America. While in the USA he taught at three USA universities and presented 15 research papers published in the proceedings of ASIS, ACM, (WDSI, Information Systems Educators Conference, The Informing Science Institute, and the International Conference on Information Quality at MIT. He is a member of ACM, DSI and a charter member of the Association for Information Systems and the Institute of Informing Science. 